Case Report

\title{
Lone Hepatocellular Carcinoma: An Isolated Chest Wall Malignancy
}

\author{
Joseph Allencherril, ${ }^{1}$ Sebastian Bruera, ${ }^{1}$ Ronan Allencherril, ${ }^{2}$ and Richard J. Hamill ${ }^{1}$ \\ ${ }^{1}$ Department of Medicine, Baylor College of Medicine, One Baylor Plaza, Houston, TX, USA \\ ${ }^{2}$ University of Texas Medical Branch, Galveston, TX, USA \\ Correspondence should be addressed to Joseph Allencherril; allenche@bcm.edu
}

Received 5 June 2017; Accepted 3 August 2017; Published 30 August 2017

Academic Editor: Isidro Machado

Copyright (C) 2017 Joseph Allencherril et al. This is an open access article distributed under the Creative Commons Attribution License, which permits unrestricted use, distribution, and reproduction in any medium, provided the original work is properly cited.

Herein we describe the case of an elderly diabetic gentleman presenting with a two-week history of dyspnea and nonproductive cough, found to have a large left anterolateral chest wall mass. Further characterization through computed tomography (CT) of the chest revealed a soft tissue mass in the left anterior lower hemithorax found to be hepatocellular carcinoma (HCC). The liver, spleen, and pancreas were unremarkable. Diagnostic labs were unremarkable. The patient had no history of hepatitis, alcohol abuse, or illicit substance use. Pathological examination and immunohistochemical staining of the chest mass biopsy were consistent with metastatic hepatocellular carcinoma (HCC). The patient opted to pursue no further medical intervention and expired two weeks later. To the authors' knowledge, this is one of very few descriptions of isolated hepatocellular carcinoma found in the absence of a primary liver lesion and classical risk factors for hepatocarcinogenesis. This case highlights that HCC may present independently of liver lesions seen on imaging in a patient without clear signs or symptoms of liver. HCC should be considered in cases of isolated tumors with unclear primaries as ectopic carcinogenesis and occult primary malignancy are possibilities.

\section{Introduction}

Hepatocellular carcinoma (HCC) is one of the most common cancers worldwide, estimated to be responsible for over one million cancer deaths annually [1]. Important risk factors for HCC include cirrhosis secondary to either chronic hepatitis $\mathrm{B}$ or hepatitis $\mathrm{C}$, obesity, diabetes, and related nonalcoholic fatty liver disease (NAFLD) [1]. It is uncommon for patients to present with HCC without the aforementioned risk factors.

The clinical presentations of HCC are highly variable. One case describes a patient presenting with oral pain and who was found to have HCC metastasized to the jaw [2]. However, even in unusual cases of HCC, it is exceedingly rare for this malignancy to present in the absence of a primary liver lesion [3, 4]. In this case, we present a patient with few overt risk factors for HCC who had an unusual presentation with no radiographic evidence of a primary tumor.

\section{Case Presentation}

An 82-year-old man with a past medical history of diabetes mellitus presented to the hospital with a two-week history of worsening shortness of breath, cough, and pain on the left anterolateral chest wall. Prior to this, the patient was only taking metformin for diabetes. He would visit his primary care physician yearly and had no previous lab abnormalities on routine examination. He was a former tobacco user but had no history of alcohol, or illicit substance use.

He was hemodynamically stable with an oxygen saturation of $90 \%$ on room air. Physical examination revealed decreased breath sounds of his left lower lung fields and exquisite tenderness to palpation of his left anterior chest wall with no crepitus. This area was mildly indurated.

Laboratory examinations showed a mild leukocytosis $(11,700 / \mu \mathrm{L})$ with normal liver enzymes and basic metabolic panel. HIV and hepatitis screening panels were negative. 


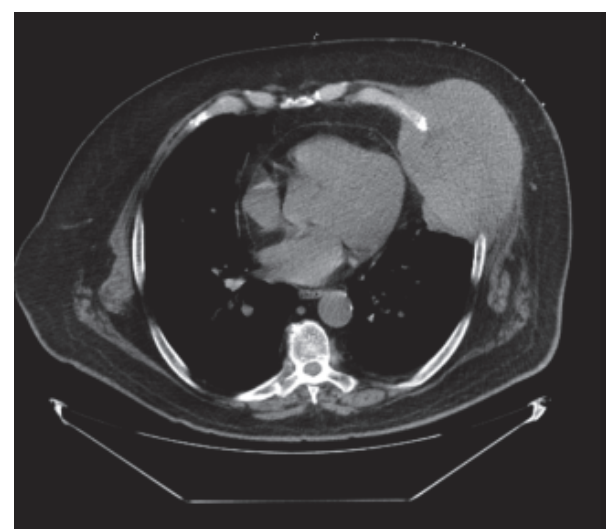

FIGURE 1: CT of chest with contrast showing $10 \times 13 \mathrm{~cm}$ soft tissue mass in the left anterior lower hemithorax, from the left anterior 6th rib to the EO muscle and left pericardium, inseparable from LV myocardium.

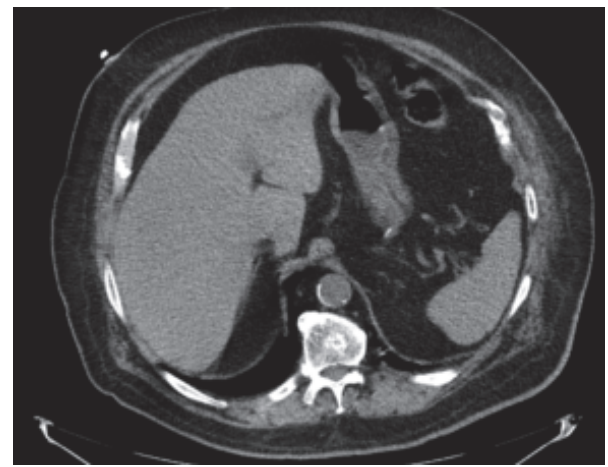

FIGURE 2: CT of abdomen with contrast showing unremarkable liver, spleen, pancreas, and adrenal glands.

A chest radiograph revealed a dense opacity at the periphery of the left lung base concerning for a mass. Further characterization through computed tomography (CT) of the abdomen and thorax with contrast demonstrated a $10 \times$ $13.3 \mathrm{~cm}$ soft tissue mass in the left anterior lower hemithorax, extending from the left anterior sixth rib to the external oblique muscle and left pericardium (Figure 1). Furthermore, there were two exophytic masses in the left upper kidney and left lower kidney, highly suspicious for renal cell carcinoma. The visualized liver, spleen, pancreas, and adrenal glands were unremarkable with no masses (Figure 2). A biopsy of the chest wall mass with subsequent microscopic pathological examination was initially suggestive of metastatic renal cell carcinoma. However, immunohistochemical stains subsequently showed oncocytic cells with endothelial cell wrapping staining positive for HepPar-1 and Glypican-3, specific markers for HCC [5] and negative for CD10, CK7, and high molecular weight cytokeratin, markers of renal cell carcinoma [6,7]. Additional immunostains showed tumor cells negative for PAX8 and a canalicular pattern with polyclonal CEA, supporting the final diagnosis of metastatic HCC in the chest wall without evidence of a primary liver source. After discussions with the patient and his family, the patient ultimately chose to not pursue further treatment or diagnostic testing for his malignancy. He opted for home hospice care given his belief in minimalist medicine.

\section{Discussion}

We have presented herein the unusual case of a gentleman with HCC presenting as a solitary lesion in the left anterolateral chest wall. On their own, chest wall tumors themselves are quite rare in the American and European populations [8].

Reports of HCC in the absence of a culprit primary liver lesion are exquisitely rare, especially as a first presentation of the disease $[3,4,9]$. HCC most commonly metastasizes to the lung, abdominal lymph nodes, and bone although extrahepatic metastasis typically occurs only in patients with advanced intrahepatic tumors [10]. Specifically, there are only a handful of cases with reported chest wall metastases of isolated HCC $[3,4,11,12]$. The pathophysiology of such presentations is poorly understood. One purported mechanism is that the remote lesions stem from the spread of a microhepatocellular carcinoma [13]. In these scenarios, it is also possible that the primary hepatic tumor may simply have escaped delineation by established imaging modalities. Spontaneous regression of an original HCC lesion is another possibility, albeit unusual and exceedingly rare as well [14]. Malignant cells may spread from the primary site while the immune system brings about the resolution of the parent lesion.

An alternative possibility is that the malignant lesion arose from ectopic hepatocellular tissue. Ectopic liver is found infrequently and has been reported to be found in structures near the liver, omentum, retroperitoneum, and thorax [3]. Ectopic liver may be more prone to carcinogenesis than native tissue given that it does not possess a normally functioning vascular or ductal system [3]. Just like physiologically normal liver, ectopic liver tissue may be infected by viruses and develop steatosis or cirrhosis. In fact, Arakawa et al. reported that only $27 \%$ of reported patients with ectopic HCC possessed a normal mother liver [15]. Asselah et al. reported the case of a gentleman with chronic hepatitis $\mathrm{C}$ infection who presented with an indolent left chest wall mass ultimately found to be solitary extrahepatic HCC [3]. The patient did well after surgical resection of the mass, and no liver lesions were found by imaging even three years later. The presentation is reminiscent of our case; however, our patient did not test positive for any infections of the liver. Of note, ectopic HCC should be distinguished from metastasis, which carries a poor prognosis.

A final explanation is that the patient had developed NAFLD with an accompanying noncirrhotic liver. In this patient, the only salient risk factor for HCC was diabetes mellitus, which is known to predispose to the development of NAFLD. Furthermore, NAFLD itself may provoke hepatic carcinogenesis even in the absence of liver cirrhosis [16]. Among the United States population, NAFLD has a prevalence of roughly $30 \%$, and the incidence of NAFLDassociated HCC has been notably increasing while the prevalence of nonalcoholic steatohepatitis (NASH) with advanced fibrosis is believed to be even higher at 66\% [17]. 
Further work is needed to clinically and pathologically distinguish ectopic liver giving rise to HCC from isolated metastases of occult HCC lesions. It is possible that patients with ectopic HCC may have better long-term prognosis [3]. Such a distinction is critical as medical or surgical intervention is usually not offered in cases of distant tumor metastases. In cases of solitary HCC metastases with an unknown primary liver lesion, it may be reasonable to pursue more aggressive imaging of the liver and even biopsy in search of an occult liver lesion in order to make this delineation. Ectopic tissue has even been found from disparate organs and systems besides the liver, including the thyroid, pancreas, adrenal glands, thymus, and neurological system [18].

This case demonstrates that HCC may present independently of clear liver lesions on radiographic imaging, even in a patient without clear signs or symptoms of liver disease or multiple risk factors for malignancy. Although it is impossible to say which particular insult may lead to hepatocarcinogenesis, a systematic approach to diagnosing and treating risk factors-such as hepatitis, illicit substance abuse, and obesity-should be adopted. We propose that clinicians encountering the exceptional cases of isolated malignant lesions without detectable lesions in the native organ, such as the liver, attempt to distinguish ectopic carcinogenesis from occult primary lesions with metastases.

\section{Conflicts of Interest}

The authors declare that they have no conflicts of interest.

\section{References}

[1] N. Muñoz and X. Bosch, "Epidemiology of hepatocellular carcinoma," in Neoplasms of the Liver, pp. 3-19, Springer, 1987.

[2] M. Pesis, S. Taicher, G. Greenberg, and A. Hirshberg, "Metastasis to the jaws as a first manifestation of hepatocellular carcinoma: Report of a case and analysis of 41 cases," Journal of Cranio-Maxillofacial Surgery, vol. 42, no. 8, pp. 1997-2001, 2014.

[3] T. Asselah, B. Condat, D. Cazals-Hatem et al., "Ectopic hepatocellular carcinoma arising in the left chest wall: a long-term follow-up," European Journal of Gastroenterology and Hepatology, vol. 13, no. 7, pp. 873-875, 2001.

[4] H.-S. Hofmann, J. Spillner, A. Hammer, and C. Diez, "A solitary chest wall metastasis from unknown primary hepatocellular carcinoma," European Journal of Gastroenterology and Hepatology, vol. 15, no. 5, pp. 557-559, 2003.

[5] D. T. Timek, J. Shi, H. Liu, and F. Lin, "Arginase-1, HepPar1 , and glypican-3 are the most effective panel of markers in distinguishing hepatocellular carcinoma from metastatic tumor on fine-needle aspiration specimens," American Journal of Clinical Pathology, vol. 138, no. 2, pp. 203-210, 2012.

[6] L. D. Truong and S. S. Shen, "Immunohistochemical diagnosis of renal neoplasms," Archives of Pathology and Laboratory Medicine, vol. 135, no. 1, pp. 92-109, 2011.

[7] M. Zhou, A. Roma, and C. Magi-Galluzzi, "The usefulness of immunohistochemical markers in the differential diagnosis of renal neoplasms," Clinics in Laboratory Medicine, vol. 25, no. 2, pp. 247-257, 2005.
[8] D. G. Cavanaugh, S. Cabellon Jr., and J. B. Peake, "A logical approach to chest wall neoplasms," Annals of Thoracic Surgery, vol. 41, no. 4, pp. 436-437, 1986.

[9] K. Horita, Y. Okazaki, A. Haraguchi, M. Natsuaki, and T. Itoh, "A case of solitary sternal metastasis from unknown primary hepatocellular carcinoma," Nihon Kyobu Geka Gakkai Zasshi, vol. 44, no. 7, pp. 959-964, 1996.

[10] S. Katyal, J. H. Oliver III, M. S. Peterson, J. V. Ferris, B. S. Carr, and R. L. Baron, "Extrahepatic metastases of hepatocellular carcinoma," Radiology, vol. 216, no. 3, pp. 698-703, 2000.

[11] Y. S. Hyun, H. S. Choi, J. H. Bae et al., "Chest wall metastasis from unknown primary site of hepatocellular carcinoma," World Journal of Gastroenterology, vol. 12, no. 13, pp. 2139-2142, 2006.

[12] S. S. Qureshi, S. V. Shrikhande, A. M. Borges, and P. J. Shukla, "Chest wall metastases from unknown primary hepatocellular carcinoma," Journal of Postgraduate Medicine, vol. 51, no. 1, pp. 41-42, 2005.

[13] A. Iosca, L. Spaggiari, and P. Salcuni, "A bone hepatocellular carcinoma metastasis without hepatic tumor: a long-term followup," American Journal of Gastroenterology, vol. 93, no. 4, article 663, 1998.

[14] R. Gómez Sanz, E. Moreno Gonzalez, F. Colina Ruiz-Delgado, H. Garcia-Muñoz, F. Ochando Cerdan, and I. Gonzalez-Pinto, "Spontaneous regression of a recurrent hepatocellular carcinoma," Digestive Diseases and Sciences, vol. 43, no. 2, pp. 323328, 1998.

[15] M. Arakawa, Y. Kimura, K. Sakata, Y. Kubo, T. Fukushima, and K. Okuda, "Propensity of ectopic liver to hepatocarcinogenesis: case reports and a review of the literature," Hepatology, vol. 29, no. 1, pp. 57-61, 1999.

[16] D. M. Torres and S. A. Harrison, "Nonalcoholic steatohepatitis and noncirrhotic hepatocellular carcinoma: fertile soil," Seminars in Liver Disease, vol. 32, no. 1, pp. 30-38, 2012.

[17] M. E. Rinella, "Nonalcoholic fatty liver disease: a systematic review," The Journal of the American Medical Association, vol. 313, no. 22, pp. 2263-2273, 2015.

[18] A. M. Marchevsky, "Lung tumors derived from ectopic tissues," Seminars in Diagnostic Pathology, vol. 12, no. 2, pp. 172-184, 1995. 


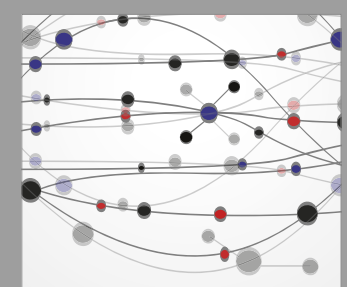

The Scientific World Journal
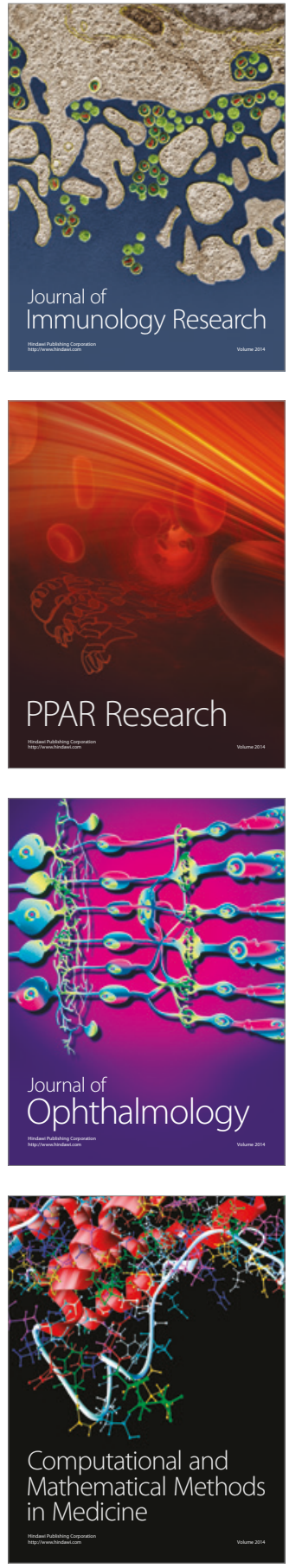

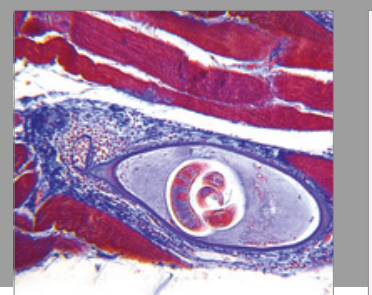

Gastroenterology Research and Practice
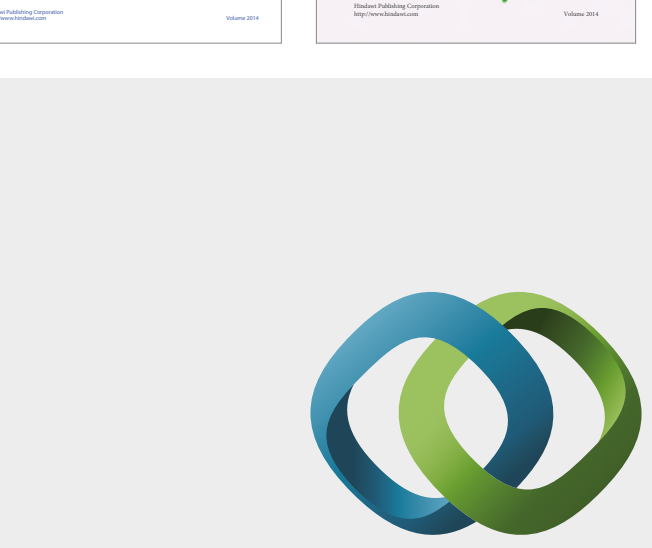

\section{Hindawi}

Submit your manuscripts at

https://www.hindawi.com
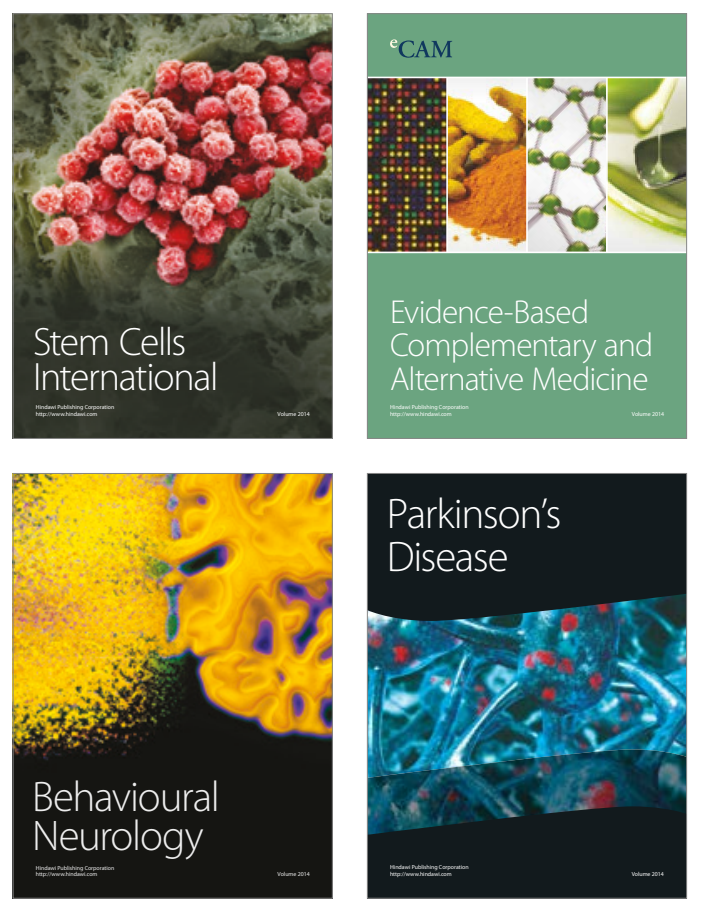
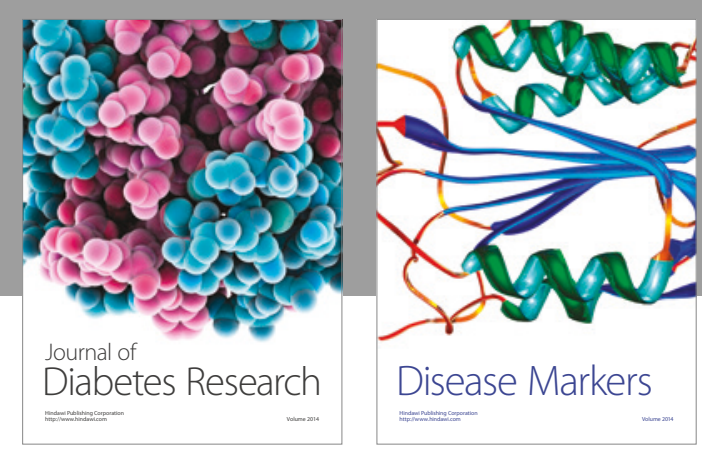

Disease Markers
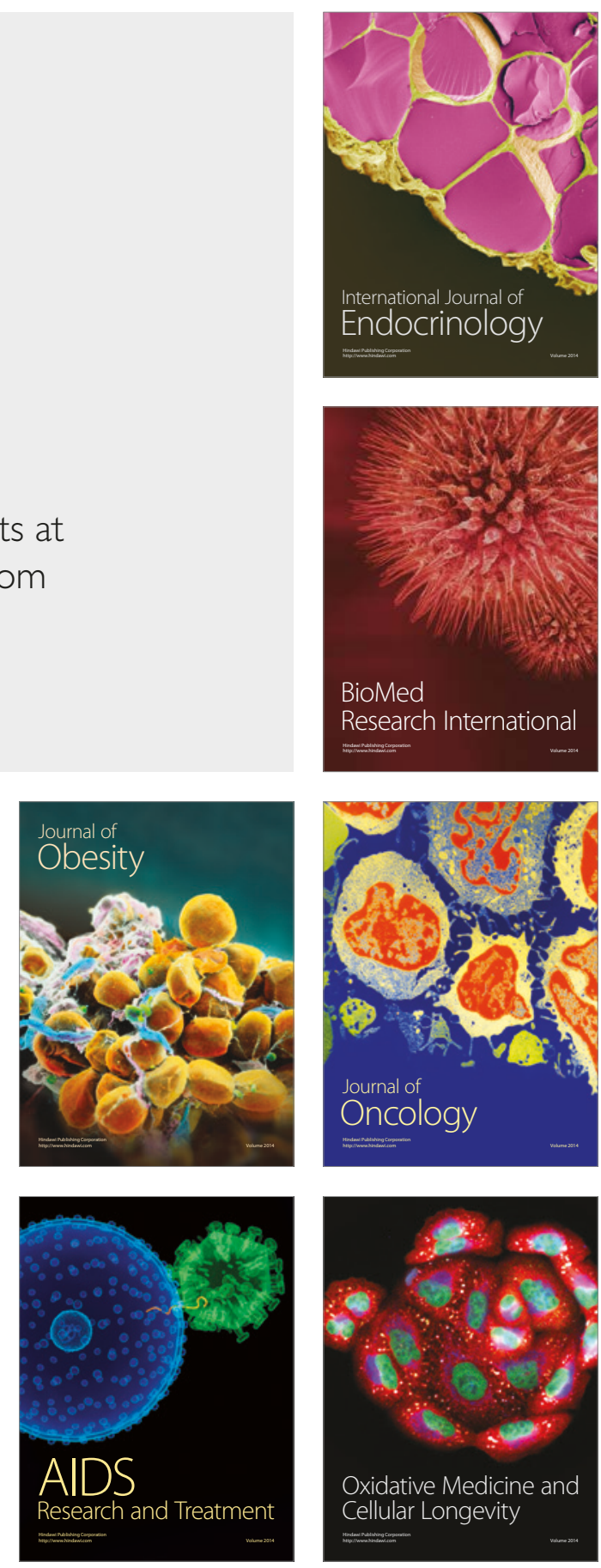